Open Access

\title{
Complete genome sequence of the thermophilic Thermus sp. CCB_US3_UF1 from a hot spring in Malaysia
}

Beng Soon Teh ${ }^{1,5^{*}}$, Nyok-Sean Lau ${ }^{1 *}$, Fui Ling Ng${ }^{2}$, Ahmad Yamin Abdul Rahman², Xuehua Wan ${ }^{3}$, Jennifer A. Saito ${ }^{3}$, Shaobin $\mathrm{Hou}^{3}$, Aik-Hong Teh${ }^{1}$, Nazalan Najimudin ${ }^{2}$ and Maqsudul Alam³,

\begin{abstract}
Thermus sp. strain CCB_US3_UF1 is a thermophilic bacterium of the genus Thermus, a member of the family Thermaceae. Members of the genus Thermus have been widely used as a biological model for structural biology studies and to understand the mechanism of microbial adaptation under thermal environments. Here, we present the complete genome sequence of Thermus sp. CCB_US3_UF1 isolated from a hot spring in Malaysia, which is the fifth member of the genus Thermus with a completely sequenced and publicly available genome (Genbank date of release: December 2, 2011). Thermus sp. CCB_US3_UF1 has the third largest genome within the genus. The complete genome comprises of a chromosome of $2.26 \mathrm{Mb}$ and a plasmid of $19.7 \mathrm{~kb}$. The genome contains 2279 protein-coding and 54 RNA genes. In addition, its genome revealed potential pathways for the synthesis of secondary metabolites (isoprenoid) and pigments (carotenoid).
\end{abstract}

Keywords: Thermus, Thermophile, Extremophile, Hot spring

\section{Introduction}

Thermus spp. are Gram-negative, aerobic, non-sporulating, and rod-shaped thermophilic bacteria. Thermus aquaticus was the first bacterium of the genus Thermus that was discovered in several of the hot springs in Yellowstone National Park, United States [1]. A few years later, two strains of Thermus thermophilus (HB27 and HB8) were successfully isolated from thermal environments in Japan $[2,3]$. To date, many strains of Thermus have been isolated from various geothermal environments such as hot springs and deep-sea hydrothermal vents. In addition to the ability to survive under thermal environments, T. thermophilus can also thrive in environments with extreme $\mathrm{pH}$ values, demonstrating great capabilities for adaptation to various environmental conditions.

The whole genome sequences of two strains of T. thermophilus, HB8 and HB27, were independently completed in 2004 [4, 5]. The genome of a second

\footnotetext{
* Correspondence: bengsoonccb@gmail.com; nyoksean@ccbusm.edu.my Deceased

${ }^{1}$ Centre for Chemical Biology, Universiti Sains Malaysia, Penang, Malaysia Full list of author information is available at the end of the article
}

Thermus species, Thermus scotoductus SA-01, is also available [6]. T. thermophilus has attracted attention as one of the model organisms for structural biology studies because protein complexes from extremophiles are easier to crystallize than their mesophilic counterparts [7]. Some of the breakthrough examples of large complexes from thermophiles that have been crystallized are structures of the $70 \mathrm{~S}$ ribosome [8], the bacterial RNA polymerase $[9,10]$ and the respiratory complex I [11] from Thermus spp. that were solved before those of Escherichia coli.

Members of the genus Thermus are of considerable biotechnological interest as sources of thermophilic enzymes [12, 13]. Thermozymes and proteins from the genus Thermus are good candidates for industrial processes because of their high thermal stability and cosolvent compatibility. The most well-known enzyme mined from the genus Thermus is DNA polymerase, an important enzyme used in PCR. Other than DNA polymerase, thermozymes from this genus are also widely used in food, pharmaceutical and paper-pulp industries [7]. Examples of industrial applications for thermostable enzymes include organic synthesis (e.g. esterases, lipases, 
proteases), starch-processing (e.g. $\alpha$-amylases, glucose isomerases), pulp and paper manufacturing (e.g. xylanases) as well as animal feed and human food production (amino acid and vitamin synthesis) [13, 14]. Here, we present a summary of classification and a set of features for Thermus sp. CCB_US3_UF1, together with the description of the complete genome sequence and annotation.

\section{Organism information}

\section{Classification and features}

Thermus spp. are suggested to be closely related to the genus Deinococcus based on several comparative studies on 16S rRNA and protein sequences, and they form a distinct branch known as the Deinococcus-Thermus group $[15,16]$. Nevertheless, the exact phylogenetic position of the Deinococcus-Thermus phylum remains to be determined. This phylum was proposed to derive from the oldest groups of the Bacteria Domain, after those of Aquifex and Thermotoga based on 16S rRNA sequence comparison [17]. A more in-depth analysis of the phylogeny of the Deinococcus-Thermus phylum based on conserved orthologs can be carried out as genome sequences from both of the genera are available [18].

In order to better understand the phylogeny of Thermus sp. CCB_US3_UF1, we constructed a phylogenetic tree based on the 16S rRNA gene sequences. There are two identical copies of the 16S rRNA gene in the Thermus sp. CCB_US3_UF1 genome. One copy of the gene sequence was used to search against the nucleotide database using NCBI BLASTN [19]. The BLASTN result shows that it has the highest sequence identity to Thermus igniterrae RF-4 (97 \%, Y18406), Thermus brockianus YS38 (96 \%, Z15062), and Thermus scotoductus Se-1 (95\%, AF032127). Figure 1 shows the phylogenetic neighborhood of Thermus sp. CCB_US3_UF1 relative to type strains of the families Deinococcaceae and Thermaceae. Chloroflexus aurantiacus (D38365) was used as an outgroup to root the tree.

Thermus sp. CCB_US3_UF1 is a Gram-negative bacterium (Table 1) and it has a rod-shaped filamentous structure (Fig. 2). Members of the genus Thermus are capable of growing at temperatures ranging between $45^{\circ} \mathrm{C}$ and $83{ }^{\circ} \mathrm{C}$ [20]. Most of them have a maximum temperature for growth at slightly below $80{ }^{\circ} \mathrm{C}[21,22]$. Interestingly, a few strains of $T$. thermophilus can grow at $80^{\circ} \mathrm{C}$ or above [23]. Thermus sp. CCB_US3_UF1 was isolated from a hot spring in Ulu Slim, Perak, Malaysia. It can grow well between $60{ }^{\circ} \mathrm{C}$ and $70{ }^{\circ} \mathrm{C}$. Thermus spp. need carbohydrates, amino acids, carboxylic acids and peptides as sources of carbon and energy. The strain CCB_US3_UF1 is an aerobic, non-sporulating, non-motile and yellow-pigmented bacterium. Some of the members of the genus Thermus are capable of growing anaerobically using nitrate as an electron acceptor and some can even reduce nitrite [22, 23].

\section{Genome sequencing and annotation}

\section{Genome project history}

The genus Thermus belongs to one of the oldest evolutionary branches of the Bacteria domain. The genome sequencing of Thermus sp. CCB_US3_UF1 was initiated as it can serve as a model bacterium for studying the

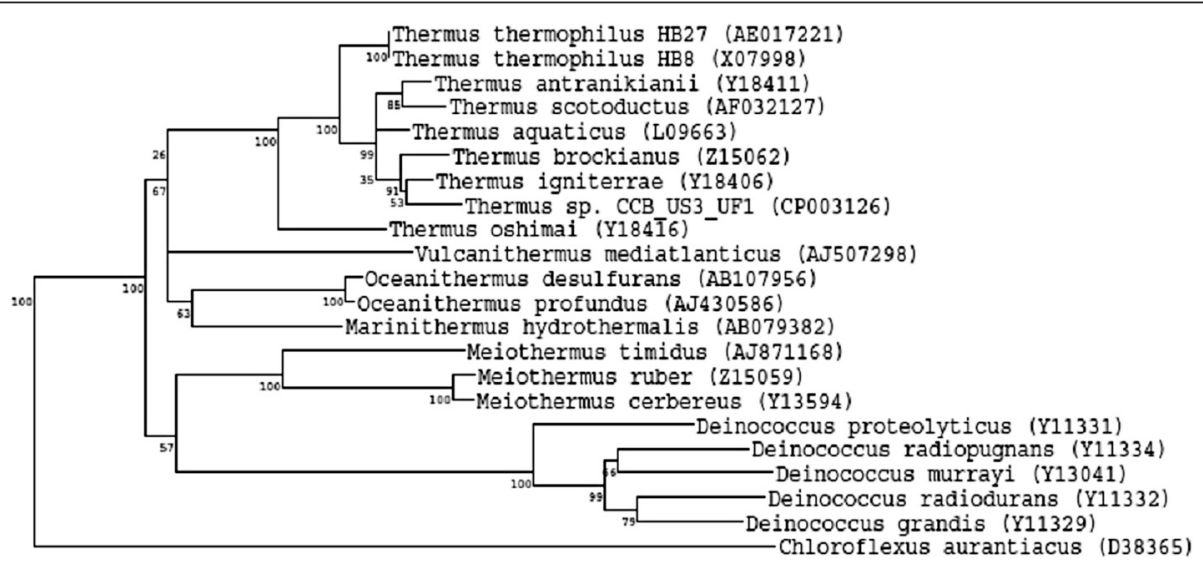

Scale:

Fig. 1 Phylogenetic tree highlighting the position of Thermus sp. CCB_US3_UF1 relative to the other type strains within the families Deinococcaceae and Thermaceae. Strains shown are those within the Deinococcaceae and Thermaceae having the corresponding NCBI genome project ids listed within [53]. The tree used sequences aligned by Ribosomal Database Project (RDP) aligner and Juke-Cantor corrected distance model. Distance matrix was constructed based on alignment model positions without the use of alignment insert, and a minimum comparable position of 200 was used. The tree was constructed with RDP Tree Builder that used Weighbor [54] with an alphabet size of 4 and a length size of 1000. The building of the tree involved a bootstrapping process that was repeated 100 times to generate a majority consensus tree [55] 
Table 1 Classification and general features of Thermus sp. CCB_US3_UF1 according to the MIGS recommendations [57]

\begin{tabular}{|c|c|c|c|}
\hline MIGS ID & Property & Term & Evidence code $^{a}$ \\
\hline & Classification & Domain Bacteria & TAS [17] \\
\hline & & Phylum Deinococcus-Thermus & TAS [58] \\
\hline & & Class Deinococci & $\operatorname{TAS}[59,60]$ \\
\hline & & Order Thermales & $\operatorname{TAS}[60,61]$ \\
\hline & & Family Thermaceae & TAS $[60,62]$ \\
\hline & & Genus Thermus & $\operatorname{TAS}[1,63,64]$ \\
\hline & & Species Unknown & IDA \\
\hline & & Type strain CCB_US3_UF1 & IDA \\
\hline & Gram stain & Negative & IDA \\
\hline & Cell shape & Rod & IDA \\
\hline & Motility & Non-motile & NAS \\
\hline & Sporulation & Non-sporulating & NAS \\
\hline & $\begin{array}{l}\text { Temperature } \\
\text { range }\end{array}$ & Thermophile $\left(45-83^{\circ} \mathrm{C}\right)$ & TAS [20] \\
\hline & $\begin{array}{l}\text { Optimum } \\
\text { temperature }\end{array}$ & $60^{\circ} \mathrm{C}$ & IDA \\
\hline & $\begin{array}{l}\text { pH range; } \\
\text { Optimum }\end{array}$ & Not reported & \\
\hline & Carbon source & Not reported & \\
\hline MIGS-6 & Habitat & Hot springs & IDA \\
\hline MIGS-6.3 & Salinity & Not-reported & \\
\hline MIGS-22 & $\begin{array}{l}\text { Oxygen } \\
\text { requirement }\end{array}$ & Aerobic & NAS \\
\hline MIGS-15 & $\begin{array}{l}\text { Biotic } \\
\text { relationship }\end{array}$ & Free-living & NAS \\
\hline MIGS-14 & Pathogenicity & Non-pathogen & NAS \\
\hline MIGS-4 & $\begin{array}{l}\text { Geographic } \\
\text { location }\end{array}$ & Ulu Slim, Perak, Malaysia & IDA \\
\hline MIGS-5 & $\begin{array}{l}\text { Sample } \\
\text { collection }\end{array}$ & 2009 & IDA \\
\hline MIGS-4.1 & Latitude & $3.898822^{\circ} \mathrm{N}$ & IDA \\
\hline MIGS-4.2 & Longitude & $101.497911^{\circ} \mathrm{E}$ & IDA \\
\hline MIGS-4.4 & Altitude & $51 \mathrm{~m}$ & IDA \\
\hline
\end{tabular}

avidence codes - IDA Inferred from Direct Assay, TAS Traceable Author Statement (i.e., a direct report exists in the literature), NAS Non-traceable Author Statement (i.e., not directly observed for the living, isolated sample, but based on a generally accepted property for the species, or anecdotal evidence). These evidence codes are from the Gene Ontology project [65]

evolution of thermophilic adaptation. The sequencing and finishing of the genome were completed at the Advanced Studies in Genomics, Proteomics and Bioinformatics (University of Hawaii) and TEDA School of Biological Sciences and Biotechnology (Nankai University, China). The genome annotation was performed at the Centre for Chemical Biology (Universiti Sains Malaysia). This genome sequence was first published in March 2012 [24]. A summary of the project information is shown in Table 2.

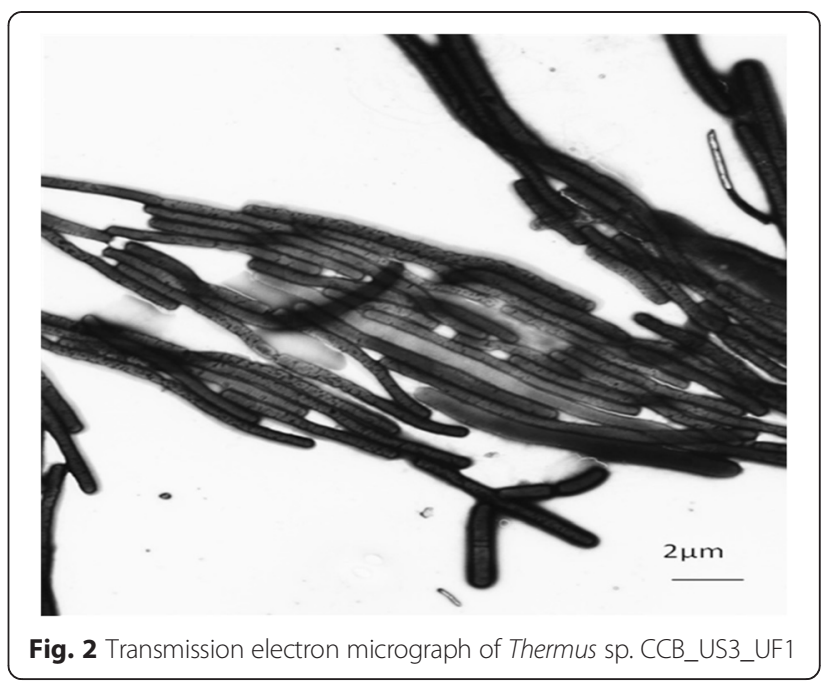

\section{Growth conditions and genomic DNA preparation}

Thermus sp. CCB_US3_UF1 was grown aerobically to late exponential phase in $50 \mathrm{ml}$ of ATCC medium 697 (Thermus medium) [3] at $60^{\circ} \mathrm{C}$. Genomic DNA was isolated from Thermus sp. CCB_US3_UF1 using a modified phenol-chloroform extraction protocol [25]. The quality of DNA was checked by $0.5 \%$ agarose gel electrophoresis and its quantity by a NanoDrop 2000 Spectrophotometer (Thermo Scientific, Wilmington, Delaware, USA). A DNA concentration of $363.4 \mathrm{ng} / \mu \mathrm{l}$ and $\mathrm{OD}_{260} / \mathrm{OD}_{280}$ of 1.90 was obtained.

Table 2 Project information

\begin{tabular}{|c|c|c|}
\hline MIGS ID & Project & Term \\
\hline MIGS 31 & Finishing quality & Finished \\
\hline MIGS-28 & Libraries used & $\begin{array}{l}\text { Two genomic libraries: one } 454 \\
\text { PE library ( } 3 \text { kb insert size), one } \\
\text { Illumina library ( } 3 \text { kb insert size) }\end{array}$ \\
\hline MIGS 29 & Sequencing platforms & $\begin{array}{l}\text { Illumina GA II×, } 454 \text { GS FLX } \\
\text { Titanium }\end{array}$ \\
\hline MIGS 31.2 & Fold coverage & $115 \times$ (Illumina); $21.14 \times(454)$ \\
\hline MIGS 30 & Assemblers & $\begin{array}{l}\text { Newbler v 2.3, burrows-wheeler } \\
\text { alignment (BWA) }\end{array}$ \\
\hline \multirow[t]{6}{*}{ MIGS 32} & Gene calling method & Glimmer 3.02 \\
\hline & Locus tag & TCCBUS3UF1 \\
\hline & Genbank ID & СР003126, СР003127 \\
\hline & GenBank date of release & December 2, 2011 \\
\hline & GOLD ID & Gp0013444 \\
\hline & BIOPROJECT & PRJN76491 \\
\hline \multirow[t]{2}{*}{ MIGS 13} & Source material identifier & CCB_US3_UF1 \\
\hline & Project relevance & $\begin{array}{l}\text { Biotechnology, pathway, } \\
\text { extremophile }\end{array}$ \\
\hline
\end{tabular}




\section{Genome sequencing and assembly}

The whole-genome sequencing of Thermus sp. CCB_US3_UF1 was performed using Roche 454 and Illumina paired-end sequencing technologies. A $3 \mathrm{~kb}$ genomic library was constructed and 97,991 paired-end reads and 54,397 single-end reads were generated using the GS FLX system, providing 21.14-fold genome coverage. Six large scaffolds including 51 contigs were successfully assembled from $97.09 \%$ of the reads using the 454 Newbler assembly software (454 Life Sciences, Branford, CT). A total of 3,469,788 reads from $3 \mathrm{~kb}$ library were produced to reach a depth of 115-fold coverage with an Illumina GA IIx (Illumina, San Diego, CA). These reads were mapped to the scaffolds using the Burrows-Wheeler Alignment (BWA) tool [26]. The majority of the gaps within the scaffolds were filled by local assembly of 454 and Illumina reads. The gaps between the scaffolds were filled by sequencing PCR products using an ABI 3730xl capillary sequencer. PCR products were sequenced to verify repeats larger than $3 \mathrm{~kb}$. The putative sequencing errors were verified and corrected by consensus of the Roche/454 and Illumina reads.

\section{Genome annotation}

The automated annotation of the genome was done using the DIYA (Do-It-Yourself Annotator) pipeline [27]. The pipeline uses Glimmer3 to predict open reading frames [28], followed by protein similarity searches using BLAST [19] against UNIREF [29], RPS-BLAST against CDD [30], and Asgard [31]. In addition, RPS-BLAST searches against the COG database was done to enable assignment of COG functional categories to the ORFs. Transfer RNAs were predicted by using tRNAscan-SE [32] while ribosomal RNAs were identified by using RNAmmer [33].

\section{Genome properties}

The complete genome of Thermus sp. CCB_US3_UF1 is composed of a single circular chromosome of 2,243,772 bp and a plasmid of 19,716 bp with $\mathrm{G}+\mathrm{C}$ contents of $68.6 \%$ and $65.6 \%$, respectively (Fig. 3). There are 2334 predicted coding sequences (CDS), 2 rRNA operons, and 48 tRNA genes in the chromosome (Table 3). A total of 32 CDS are predicted in the plasmid. The distribution of genes into COG functional categories is presented in Table 4.

\section{Comparison with other sequenced genomes}

The genome of Thermus sp. CCB_US3_UF1 (2.26 Mb) is larger than those of $T$. thermophilus HB27 (2.13 Mb) and T. thermophilus HB8 (2.12 Mb), but smaller than that of T. scotoductus SA-01 (2.36 Mb) (Table 5).

The Thermus sp. CCB_US3_UF1 genome was compared against closely related Thermus genomes using BLAST and Artemis comparison tool to identify regions of synteny. The three closest Thermus with sequenced genomes (T. thermophilus strains HB27, HB8 and T. scotoductus SA-01) were selected for the comparison. The genome of strain HB27 consists of a chromosome $(1.89 \mathrm{Mb})$ and a megaplasmid $(0.23 \mathrm{Mb})$. On the other hand, strain HB8 has a chromosome of $1.85 \mathrm{Mb}$, a megaplasmid $(0.26 \mathrm{Mb})$ and a plasmid $(9.3 \mathrm{~kb})$ [5]. The genome of T. scotoductus includes a $2.3 \mathrm{Mb}$ chromosome and a plasmid of $8.4 \mathrm{~kb}$.

Thermus sp. CCB_US3_UF1, T. thermophilus HB27, HB8 and T. scotoductus SA-01 all have a small genome size that is below $2.5 \mathrm{Mb}$. They also display a high $\mathrm{G}+\mathrm{C}$ content that may correlate with their thermophilic lifestyle. CCB_US3_UF1 has a higher number of predicted protein coding sequences (2279) than HB27 (2210) and HB8 (2173), but lower than that of T. scotoductus SA-01 (2458). They also share a similar number of rRNA (16S-23S-5S) operons with a wellbalanced high $\mathrm{G}+\mathrm{C}$ content above $60 \%$, a common feature displayed by thermophilic bacteria. The number of tRNAs that are present in all four genomes is between 47 and 48. In terms of transposase genes, $T$. scotoductus SA-01 has the highest number (22 genes), followed by CCB_US3_UF1 (13 genes), T. thermophilus HB27 (18 genes), and HB8 (18 genes). Interestingly, no prophage-related genes are found in these four genomes, implying the occurrence of clustered regularly interspaced short palindromic repeats (CRISPRs). CRISPR is characterized as a type of antiviral immune system found in Bacteria and Archaea [34].

There are 1728 proteins, or $76 \%$ of the total proteins, from Thermus sp. CCB_US3_UF1 that are found orthologous to the proteins in T. thermophilus HB27, and a total of 1691 (74 \%) orthologs are shared between CCB_US 3_UF1 and T. thermophilus HB8. Meanwhile, a total of 1885 (83 \%) proteins are shared between CCB_US3_UF1 and T. scotoductus SA-01, showing greater similarity between these two species. The protein ortholog mapping was done with a cut-off e-value of $10^{-5}$ using the proteinprotein BLAST (blastp). Despite of the similarity of many of their gene products, genome-wide synteny between Thermus sp. CCB_US3_UF1 and T. thermophilus HB27, HB8 and T. scotoductus SA-01 could not be detected.

The plasmid of Thermus sp. CCB_US3_UF1 shows no overall similarity to the other sequenced plasmids of $T$. thermophilus HB27 and HB8, but it has high similarity to the plasmid of Thermus sp. 4C, designated as pL4C [35]. The gene encoding chromosome segregation ATPase (TCCBUS3UF1_p160) that is found in pL4C is present in the Thermus sp. CCB_US3_UF1 plasmid. This protein has been suggested to play an essential role in plasmid replication and partition [36]. In addition, a putative integrase gene that facilitates gene transfer and chromosome modification can be found in both plasmids. 


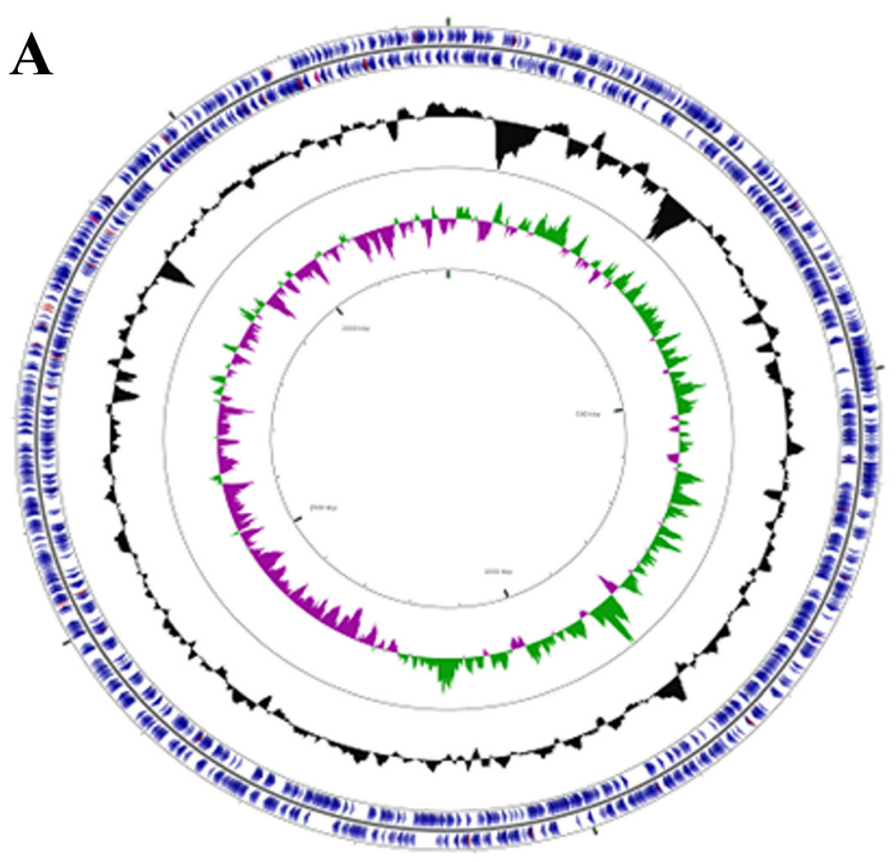

B

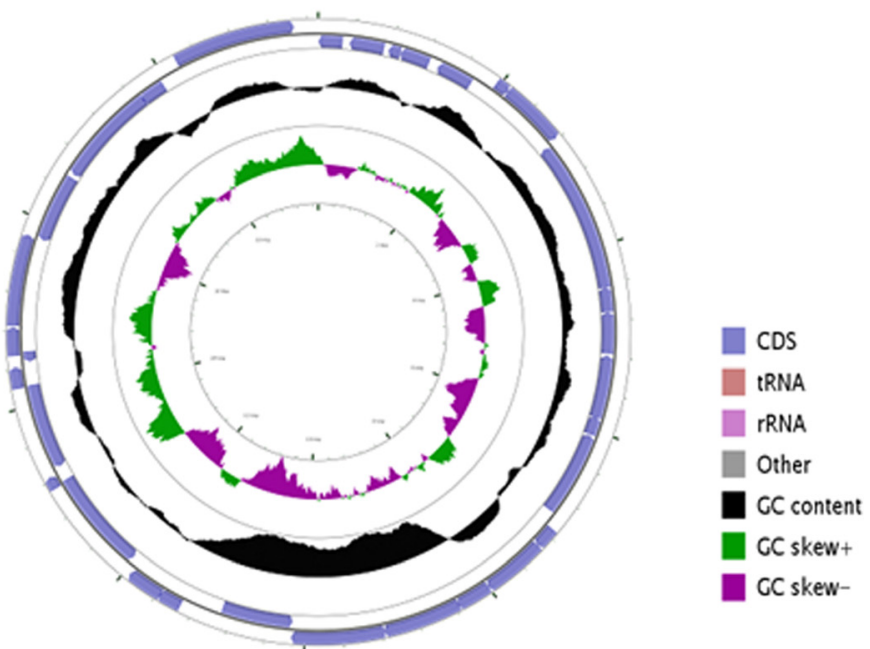

Fig. 3 Graphical circular map of the Thermus sp. CCB_US3_UF1 chromosome and plasmid PTCCB09. a Chromosome. b Plasmid. From the inside to outside, the second and fourth circles show GC skew and $\mathrm{G}+\mathrm{C}$ content respectively. The sixth and seventh circles show protein coding genes in positive and negative strands and RNA genes (tRNAs red, rRNAs light purple, other RNAs grey). This figure was generated by CGView [56]

\section{Insights from the genome sequence}

The Thermus sp. CCB_US3_UF1 genome encodes genes for complete tricarboxylic cycle, gluconeogenesis, glyoxylate bypass and Embden-Meyerhof pathways. Both Thermus sp. CCB_US3_UF1 and T. thermophilus HB27 share similar sets of genes that are involved in aerobic respiration. At high temperatures, the solubility of oxygen in water is low. Two terminal cytochrome oxidases are found

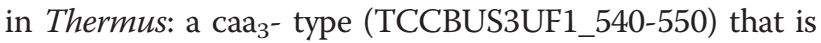
expressed under high oxygen levels, and a $\mathrm{ba}_{3}$ - type oxidase (TCCBUS3UF1_13990, TCCBUS3UF1_14010) that is expressed under low oxygen supply $[37,38]$. Thermus sp. CCB_US3_UF1 is able to synthesize many important compounds, including amino acids, vitamins, cofactors, carriers, purines and pyrimidines. Many of these biosynthetic pathways show a high degree of conservation between CCB_US3_UF1 and T. thermophilus HB27. In addition, Thermus sp. CCB_US3_UF1 has branched-chain amino acid $\mathrm{ABC}$ transport systems that are important for nutrient acquisition, and ion transporters for the elimination of toxic compounds such as copper and arsenite. 
Table 3 Genome statistics

\begin{tabular}{llr}
\hline Attribute & Value & \% of Total $^{a}$ \\
\hline Genome size (bp) & $2,263,488$ & 100.00 \\
DNA coding (bp) & $2,137,656$ & 94.44 \\
DNA G + C (bp) & $1,552,285$ & 68.58 \\
DNA scaffolds & 1 & 100.00 \\
Total genes ${ }^{\text {b }}$ & 2,333 & 100.00 \\
Protein coding genes & 2,279 & 97.64 \\
RNA genes & 54 & 2.31 \\
Pseudo genes & 1 & 0.04 \\
Genes in internal clusters & 822 & 36.07 \\
Genes with function prediction & 2,072 & 90.92 \\
Genes assigned to COGs & 2,098 & 89.89 \\
Genes with Pfam domains & 1,469 & 64.46 \\
Genes with signal peptides & 113 & 4.96 \\
Genes with transmembrane helices & 460 & 20.18 \\
CRISPR repeats & 8 & 0.34 \\
\hline
\end{tabular}

${ }^{a}$ The total is based on either the size of the genome in base pairs or the total number of protein coding genes in the annotated genome

${ }^{b}$ Pseudogenes may also be counted as protein coding or RNA genes, so their number is not additive under the total gene count

\section{Motility and natural transformation}

So far, motility is not observed in Thermus and no flagella biosynthetic gene is present in the genomes. However, genes encoding gliding motility proteins (TCCBUS3U F1_13970, TCCBUS3UF1_13980) and a twitching mobility protein (PilT, TCCBUS3UF1_9080) are found in the genome of Thermus sp. CCB_US3_UF1. These two proteins are also found in T. thermophilus HB27, HB8 and T. scotoductus SA-01, and this raises the question regarding the existence of motility in Thermus.

Thermus sp. CCB_US3_UF1 is also found to possess type IV pili that are crucial in the attachment, twitching motility, surface colonization, and natural transformation systems in bacteria [39]. The efficiency of a DNA uptake system in T. thermophilus is crucial to thermoadaptation and exchange of genetic materials in high temperature environments. Competence proteins play an important role in natural transformation and can be categorized into three groups: DNA-translocator-specific proteins, type IV pili (Tfp)-related proteins, and nonconserved proteins [40]. Genes encoding DNA-translocator-specific proteins [ComEA (TCCBUS3UF1_22560), ComEC (TCCBU S3UF1_22570), DprA (TCCBUS3UF1_18680)] and Tfprelated proteins [PilA1 (TCCBUS3UF1_8740), PilA2 (TC CBUS3UF1_8720), PilA3 (TCCBUS3UF1_8710)] were found in the Thermus sp. CCB_US3_UF1 genome. Genes encoding leader peptidase (PilD, TCCBUS3UF1_20930), traffic-NTPase (PilF, TCCBUS3UF1_21340), inner membrane protein (PilC, TCCBUS3UF1_8100), PilM-homolog and secretin-like protein (PilQ, TCCBUS3UF1_6320) were
Table 4 Number of genes associated with general COG

functional categories

\begin{tabular}{llrl}
\hline Code & Value & \% age & Description \\
\hline A & 147 & 6.4 & Translation, ribosomal structure and biogenesis \\
K & 23 & 1.0 & RNA processing and modification \\
L & 97 & 4.2 & Transcription \\
B & 115 & 5.0 & Replication, recombination and repair \\
D & 38 & 1.6 & Cell cycle control, cell division, chromosome \\
& & & partitioning \\
Y & 0 & 0.0 & Nuclear structure \\
V & 27 & 1.2 & Defense mechanisms \\
T & 77 & 3.3 & Signal transduction mechanisms \\
M & 91 & 3.9 & Cell wall/membrane biogenesis \\
N & 63 & 2.7 & Cell motility \\
Z & 0 & 0.0 & Cytoskeleton \\
W & 0 & 0.0 & Extracellular structures \\
U & 50 & 2.2 & Intracellular trafficking and secretion \\
O & 90 & 3.9 & Posttranslational modification, protein turnover, \\
& & & chaperones \\
C & 145 & 6.3 & Energy production and conversion \\
G & 138 & 6.0 & Carbohydrate transport and metabolism \\
E & 247 & 10.7 & Amino acid transport and metabolism \\
F & 71 & 3.1 & Nucleotide transport and metabolism \\
H & 115 & 5.0 & Coenzyme transport and metabolism \\
I & 95 & 4.1 & Lipid transport and metabolism \\
P & 95 & 4.1 & Inorganic ion transport and metabolism \\
Q & 56 & 2.4 & Secondary metabolites biosynthesis, transport \\
& & & and catabolism \\
R & 310 & 13.4 & General function prediction only \\
S & 215 & 9.3 & Function unknown \\
\hline & 181 & 7.8 & Not in CoGs \\
\hline & & & \\
& & &
\end{tabular}

${ }^{a}$ The total is based on the total number of protein coding genes in the genome

also identified. In addition, genes encoding competence proteins ComZ (TCCBUS3UF1_870), PilN (TCCBUS3UF 1_6350), PilO (TCCBUS3UF1_6340), and PilW (TCCBUS3 UF1_6330) were present in the chromosome of CCB_US 3_UF1. The genes encoding PilM, PilN, PilO, PilW, and PilQ are found to cluster together in the genome (Fig. 4). The rearrangement of these genes is different in Thermus sp. CCB_US3_UF1 compared to T. thermophilus HB27, HB8 and T. scotoductus SA-01, demonstrating the loss of synteny between CCB_US3_UF1 and the other Thermus bacteria. The involvement of Thermus pili in DNA uptake has yet to be determined.

\section{Genomic islands}

Potential genomic islands present in the Thermus sp. CCB_US3_UF1 genome were predicted using the 
Table 5 Comparison of genome features of different species of Thermus

\begin{tabular}{lllll}
\hline Species & Thermus sp. CCB_US3_UF1 & Thermus thermophilus HB27 & Thermus thermophilus HB8 & Thermus scotoductus SA-01 \\
\hline Genome size (bp) & $2,263,488$ & $2,127,482$ & $2,116,056$ & $2,355,186$ \\
G+C content (\%) & 68.6 & 69.4 & 69.5 & 64.9 \\
Number of protein coding genes & 2,279 & 2,210 & 2,173 & 2,458 \\
Coding area (\%) & 94.4 & 94.8 & 94.9 & 94.0 \\
Total number of genes & 2,333 & 2,263 & 2,226 & 2,511 \\
Hypothetical genes & 742 & 734 & 758 & 619 \\
Proteins with assigned function & 1,537 & 1,476 & 1,415 & 1,839 \\
rRNA & 6 & 6 & 6 & 6 \\
tRNA & 48 & 47 & 47 & 47 \\
Transposase & 13 & 18 & 18 & 22 \\
CRISPR sequences & 8 & 10 & 11 & 3 \\
\hline
\end{tabular}

Table adapted from NCBI

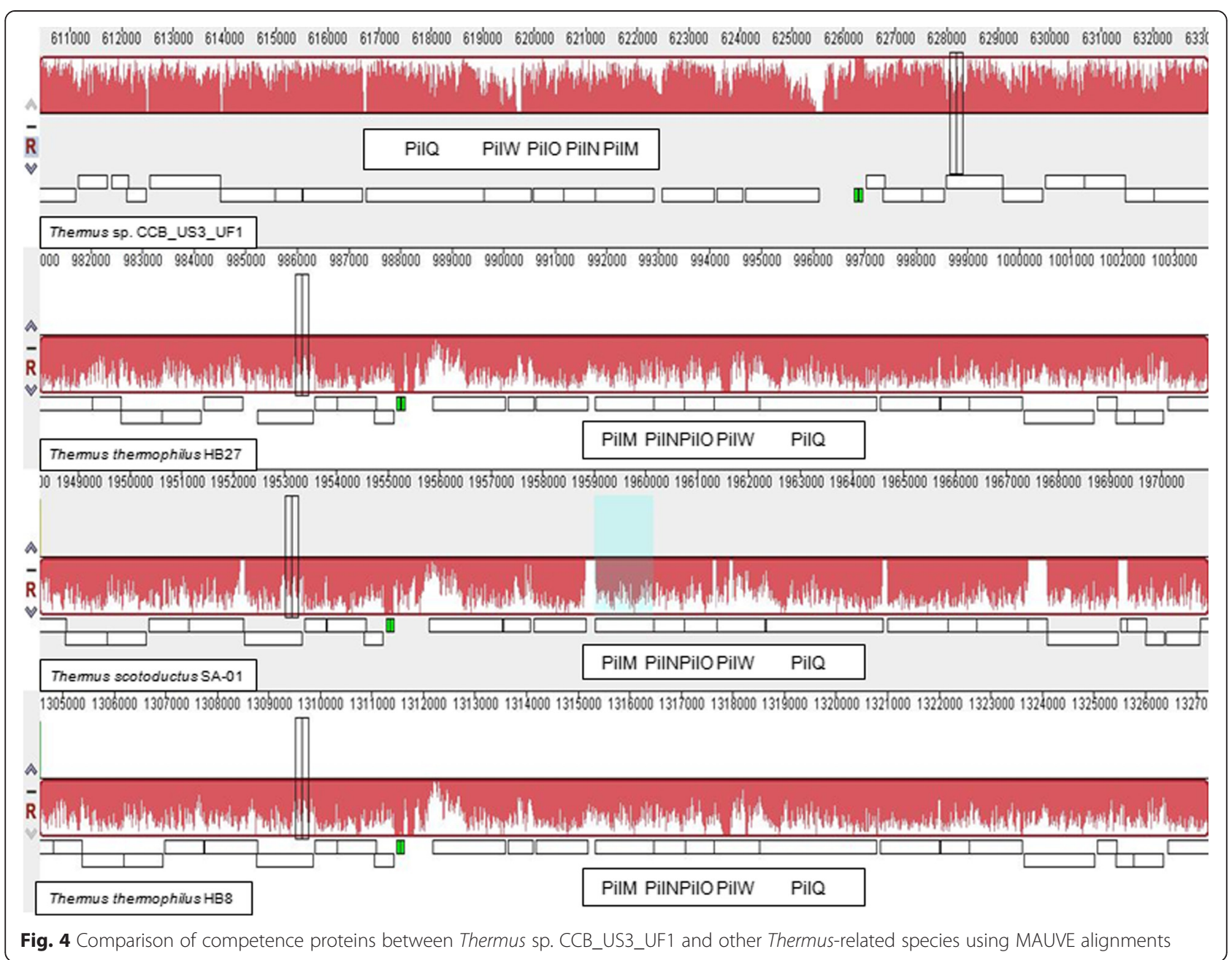


IslandViewer database [41]. Early studies on genomic islands focused on regions that carry virulence factors and they are termed pathogenicity islands. Genomic islands are also shown to carry various types of genes associated with many metabolic pathways or biological processes [42]. A total of 11 possible genomic islands were identified in the Thermus sp. CCB_US3_UF1 genome. Several of these genomic islands carry genes encoding proteins involved in transport systems and defense mechanisms. For example, genomic islands 2, 3, 6 and 7 contain numerous transporter genes that may be involved in membrane transport in Thermus sp. CCB_US3_UF1. It is interesting to note that CRISPR-associated Cas proteins that are associated with phage immunity are present on Genomic Island 8 (245986 - 276477) and Genomic Island 10 (1323615 - 1334721). In comparison with other members of Thermus, T. thermophilus HB27 harbors 10 genomic islands while both T. thermophilus HB8 and T. scotoductus SA-01 carry 13 genomic islands.

\section{CRISPR}

CRISPR is an RNAi-like system that provides adaptive immunity against phages or other infections is present in prokaryotes [43]. Using the CRISPR Finder tool [44], eight CRISPR repeat regions were detected in the Thermus sp. CCB_US3_UF1 genome (Table 6). The number of spacers in each of these loci are 3, 17, 14, 23, $18,9,12$, and 2 respectively, i.e. a total of 98 spacers.

A comparison with other Thermus sp. revealed that a total of 10, 11 and 3 CRISPRs were found in T. thermophilus HB27, HB8 and T. scotoductus SA-01, respectively. In terms of the number of spacers, T. thermophilus HB8 has the largest (112), followed by Thermus sp. CCB_US3_UF1 (98), T. scotoductus SA-01 (87) and T. thermophilus HB27 (74). The existence of a large number of CRISPRs in the Thermus genomes reflects an adaptation strategy employed by Thermus to protect themselves from foreign DNA invasion from the surrounding environments.

Table 6 Direct repeat consensus sequences of CRISPR loci

\begin{tabular}{ll}
\hline CRISPR locus & Direct repeat consensus \\
\hline 1 & GTAGTCCCCACGCACGTGGGGATGGACC \\
2 & GTITCAAACCCTCATAGGTACGGTCAGAAC \\
3 & CTITGAACCGTACCTATAAGGGTTGAAAC \\
4 & CTITGAACCGTACCTATAAGGGTTGAAAC \\
5 & GTTGCAAAAGTGGTTCCCCGCAAGGGGTTGCGAC \\
6 & GTCGCAATCCCCTTACGGGGAGCCACTTTGCAAC \\
7 & GTCGCAATCCCCTTACGGGGAAGCACTTTGCAAC \\
8 & CGTAGTCCCCACACGCGTGGGGATGGACC \\
\hline
\end{tabular}

\section{Isoprenoid biosynthesis}

Based on the genome information, Thermus sp. CCB_US 3_UF1 synthesizes precursors for isoprenoid compounds from pyruvate and glyceraldehyde 3-phosphate using the deoxyxylulose phosphate (MEP/DOXP) pathway instead of the mevalonate pathway. Isoprenoid compounds are derived from the five-carbon precursor isopentenyl diphosphate (IPP). The genes encoding enzymes of the complete DOXP pathway could be identified in the genome. The DOXP pathway is initiated by the conversion of glyceraldehyde 3-phosphate and pyruvate to 1-deoxy-D-xylulose 5-phosphate (DOXP) catalyzed by DOXP synthase (TCCBUS3UF1_200). Isoprenoid synthesis then proceeds through a series of enzymatic reactions that lead to the formation of 2-C-methyl-D-erythritol2,4-cyclodiphosphate. Genes encoding the enzymes involved are $d x r$ (TCCBUS3UF1_15410), ispD (TCCBUS3 UF1_19830), ispE (TCCBUS3UF1_19820), and ispF (TCC BUS3UF1_380). Genes $g c p E$ (TCCBUS3UF1_18880) and lytB (TCCBUS3UF1_22000), which encode the enzymes involved in the last two steps of isoprenoid synthesis that lead to IPP formation are also encoded in the genome (Additional file 1: Figure S1).

\section{Carotenoid biosynthesis}

Most species of the genus Thermus are characterized by the ability to synthesize yellow carotenoid-like pigments [45]. Carotenoids are natural pigments that have been used commercially as food colorants, nutrient supplements and for pharmaceuticals purposes [46]. T. thermophilus has been shown to produce carotenoids known as thermozeaxanthins and thermobiszeaxanthins [47]. As carotenoids are one of the hydrophobic components associated with the cell membrane, it was suggested that carotenoids might have an essential role in stabilizing the membrane of Thermus at high temperature. In $T$. thermophilus HB27, genes encoding the terminal steps of carotenoid biosynthesis are found in the large plasmid (pTT27), whereas precursor synthesis involving the formation of geranylgeranyl pyrophosphate (GGPP) is accomplished by enzymes encoded on the chromosome [5]. In Thermus sp. CCB_US3_UF1, genes encoding the enzymes for both the terminal and precursor steps of carotenoid biosynthesis are located on the chromosome (Additional file 2: Figure S2).

In the bacterial carotenoid biosynthetic pathway, phytoene is the first carotenoid synthesized and it is formed from the condensation of two molecules of geranylgeranyl pyrophosphate (GGPP) [48]. The GGPP synthase gene (TTHA0013) from T. thermophilus HB8 has been identified and functionally characterized [49]. The gene has a homolog (TCCBUS3UF1_18840) in Thermus sp. CCB_US3_UF1. Phytoene is synthesized from GGPP by phytoene synthase (CrtB). In T. thermophilus 
HB27, a gene encoding a homolog of phytoene synthase (TT_P0057) was cloned and identified as $c r t B$. It was suggested that phytoene synthase is the rate-limiting enzyme in the carotenoid biosynthesis in $T$. thermophilus. In addition, $c r t B$ of $T$. thermophilus was found to cluster together with other carotenogenic genes on the large plasmid [50]. Interestingly, the homolog of $\mathrm{crtB}$ (TCCBUS 3UF1_10160) in Thermus sp. CCB_US3_UF1 is encoded on the chromosome and not the plasmid. Phytoene is then converted to lycopene via a series of desaturation steps that are catalyzed by phytoene desaturase $(\mathrm{CrtI})$, cis-carotene isomerase $(\mathrm{CrtH})$ and $\zeta$-carotene desaturase [51]. In Deinococcus-Thermus bacteria, only one phytoene desaturase, CrtI, has been detected. A gene encoding a CrtI homolog (TCCBUS3UF1_10090) is detected in the genome of Thermus sp. CCB_US3_UF1 as well $[5,52]$. Following lycopene synthesis, the carotenoid biosynthetic pathway branches into acyclic and cyclic carotenoids formation. A possible gene encoding an enzyme that catalyzes the cyclization of lycopene, CrtY-type lycopene cyclase (TCCBUS3UF1_10120) is found in the genome of Thermus sp. CCB_US3_UF1 and the other three sequenced Thermus genomes [52] (Additional file 2: Figure S2).

\section{Conclusion}

Thermus have proven to be useful as sources of thermostable enzymes and the genome sequences provide information for further exploring the biotechnological potentials of this genus. Analysis of the Thermus sp. CCB_US3_UF1 genome revealed that it encodes pathways for the synthesis of secondary metabolites (isoprenoid) and pigments (carotenoid). The latter has attracted industrial interest for application in food industries. The CRISPR/ Cas system that is found in Thermus could be an interesting tool in molecular biology, particularly for genome editing. Considering the great potential of Thermus in various fields, the complete genome sequence of Thermus sp. CCB_US3_UF1 is a valuable resource for both fundamental researches and biotechnological applications.

\section{Additional files}

Additional file 1: Figure S1. Metabolic pathway reconstruction of isoprenoid biosynthesis. (TIFF $2139 \mathrm{~kb}$ )

Additional file 2: Figure S2. Metabolic pathway reconstruction of carotenoid biosynthesis. (TIFF $2137 \mathrm{~kb}$ )

\author{
Abbreviations \\ CCB: Centre for chemical biology; TEDA: Tianjin economic-technological \\ developmental area; ATCC: American type culture collection; \\ CRISPR: Clustered regularly interspaced short palindromic repeats; \\ BWA: Burrows-wheeler alignment; USM: Universiti Sains Malaysia.
}

\section{Competing interests}

The authors declare that they have no competing interests.

\section{Authors' contributions}

MA and NN conceived the project. SH and XW performed library preparation, genome sequencing and assembly. AYAR worked on genome annotation. BST and FLN performed pathway analysis. BST worked on genome analysis and comparative genomics. NSL, JAS and AHT were involved in editing and critical revision of the manuscript. BST prepared and wrote the manuscript. All authors read and approved the final manuscript.

\section{Acknowledgements}

We would like to gratefully acknowledge the help of Dr. Rashidah Abdul Rahim and Muhd Izar bin Omar for isolating the pure culture of Thermus sp. CCB_US3_UF1. This work was partially supported by USM funding 1001/ PCCB/870009 to the Centre for Chemical Biology, Universiti Sains Malaysia. This paper is dedicated to our mentor and colleague Maqsudul Alam, in memory of his contributions to genome science.

\section{Author details}

${ }^{1}$ Centre for Chemical Biology, Universiti Sains Malaysia, Penang, Malaysia. ${ }^{2}$ School of Biological Sciences, Universiti Sains Malaysia, Penang, Malaysia. ${ }^{3}$ Advanced Studies in Genomics, Proteomics and Bioinformatics, University of Hawaii, Honolulu, Hawaii, USA. ${ }^{4}$ Department of Microbiology, University of Hawaii, Honolulu, Hawaii, USA. ${ }^{5}$ Present address: Department of Bioorganic Chemistry, Max Planck Institute for Chemical Ecology, Jena, Germany.

Received: 27 October 2014 Accepted: 28 July 2015

Published online: 08 October 2015

\section{References}

1. Brock TD, Freeze H. Thermus aquaticus gen. n. and sp. n., a nonsporulating extreme thermophile. J Bacteriol. 1969;98(1):289-97.

2. Williams RA, Smith KE, Welch SG, Micallef J, Sharp RJ. DNA relatedness of Thermus strains, description of Thermus brockianus sp. nov., and proposal to reestablish Thermus thermophilus (Oshima and Imahori). Int I Syst Bacteriol. 1995;45(3):495-9.

3. Oshima T, Imahori K. Thermus thermophilus (Yoshida and Oshima) comb. nov., a non-sporulating thermophilic bacterium from a Japanese thermal spa. Int J Syst Bacteriol. 1974;24:102-12.

4. Masui R, Kurokawa K, Nakagawa N, Tokunoga F, Koyama Y, Shibata T et al. Thermus thermophilus HB8, complete genome. NCBI. 2005.

5. Henne A, Bruggemann H, Raasch C, Wiezer A, Hartsch T, Liesegang H, et al. The genome sequence of the extreme thermophile Thermus thermophilus. Nat Biotechnol. 2004;22(5):547-53.

6. Gounder K, Brzuszkiewicz E, Liesegang H, Wollherr A, Daniel R, Gottschalk G, et al. Sequence of the hyperplastic genome of the naturally competent Thermus scotoductus SA-01. BMC Genomics. 2011;12:577.

7. Cava F, Hidalgo A, Berenguer J. Thermus thermophilus as biological model. Extremophiles. 2009;13(2):213-31.

8. Yusupov MM, Yusupova GZ, Baucom A, Lieberman K, Earnest TN, Cate JH, et al. Crystal structure of the ribosome at $5.5 \mathrm{~A}$ resolution. Science. 2001;292(5518):883-96.

9. Severinov K. RNA polymerase structure-function: insights into points of transcriptional regulation. Curr Opin Microbiol. 2000;3(2):118-25.

10. Selmer M, Dunham CM, Murphy FV, Weixlbaumer A, Petry S, Kelley AC, et al. Structure of the 70 ribosome complexed with mRNA and tRNA. Science. 2006;313(5795):1935-42.

11. Sazanov LA, Hinchliffe P. Structure of the hydrophilic domain of respiratory complex I from Thermus thermophilus. Science. 2006;311(5766):1430-6.

12. Pantazaki AA, Pritsa AA, Kyriakidis DA. Biotechnologically relevant enzymes from Thermus thermophilus. Appl Microbiol Biotechnol. 2002;58(1):1-12.

13. Niehaus F, Bertoldo C, Kahler M, Antranikian G. Extremophiles as a source of novel enzymes for industrial application. Appl Microbiol Biotechnol. 1999;51(6):711-29.

14. Vieille C, Zeikus GJ. Hyperthermophilic enzymes: sources, uses, and molecular mechanisms for thermostability. Microbiol Mol Biol Rev. 2001;65(1):1-43.

15. Omelchenko MV, Wolf YI, Gaidamakova EK, Matrosova VY, Vasilenko A, Zhai $\mathrm{M}$, et al. Comparative genomics of Thermus thermophilus and Deinococcus radiodurans: divergent routes of adaptation to thermophily and radiation resistance. BMC Evol Biol. 2005;5:57. 
16. Griffiths E, Gupta RS. Identification of signature proteins that are distinctive of the Deinococcus-Thermus phylum. Int Microbiol. 2007;10(3):201-8.

17. Woese CR. Bacterial evolution. Microbiol Rev. 1987;51(2):221-71.

18. Ciccarelli FD, Doerks T, von Mering C, Creevey CJ, Snel B, Bork P. Toward automatic reconstruction of a highly resolved tree of life. Science. 2006:311(5765):1283-7.

19. Altschul SF, Gish W, Miller W, Myers EW, Lipman DJ. Basic local alignment search tool. J Mol Biol. 1990;215(3):403-10.

20. da Costa MS, Rainey FA, Nobre MF. The Genus Thermus and Relatives. In: The Prokaryotes: a Handbook on the Biology of Bacteria, vol. 7. 3rd ed. 2006. p. 797-812.

21. da Costa MS, M. F. Nobre, Rainey FA. The genus Thermus. In: D R Boone and R W Castenholz (Eds) Bergey's Manual of Systematic Bacteriology, 2nd ed Springer-Verlag New York, NY. 2001b;1:404-14.

22. Chung AP, Rainey FA, Valente M, Nobre MF, da Costa MS. Thermus igniterrae sp. nov. and Thermus antranikianii sp. nov., two new species from Iceland. Int J Syst Evol Microbiol. 2000;50(Pt 1):209-17.

23. Manaia CM, Hoste B, Gutierrez MC, Gillis M, Ventosa A, Kersters K, et al. Halotolerant Thermus strains from marine and terrestrial hot springs belong to Thermus thermophilus (ex Oshima and Imahori, 1974) nom. rev. emend. Syst Appl Microbiol. 1995;17:526-32.

24. Teh BS, Abdul Rahman AY, Saito JA, Hou S, Alam M. Complete genome sequence of the thermophilic bacterium Thermus sp. strain CCB_US3_UF1. J Bacteriol. 2012;194(5):1240.

25. Fulton J, Douglas T, Young AM. Isolation of viruses from high temperature environments. Methods Mol Biol. 2009;501:43-54.

26. Li H, Durbin R. Fast and accurate short read alignment with Burrows-Wheeler transform. Bioinformatics. 2009;25(14):1754-60.

27. Stewart AC, Osborne B, Read TD. DIYA: a bacterial annotation pipeline for any genomics lab. Bioinformatics. 2009;25:962-3.

28. Delcher AL, Bratke KA, Powers EC, Salzberg SL. Identifying bacterial genes and endosymbiont DNA with Glimmer. Bioinformatics. 2007;23(6):673-9.

29. Suzek BE, Huang H, McGarvey P, Mazumder R, Wu CH. UniRef: comprehensive and non-redundant UniProt reference clusters. Bioinformatics. 2007;23(10):1282-8.

30. Marchler-Bauer A, Lu S, Anderson JB, Chitsaz F, Derbyshire MK, DeWeese-Scott C, et al. CDD: a Conserved Domain Database for the functional annotation of proteins. Nucleic Acids Res. 2011;39(Database issue):D225-9.

31. Alves JM, Buck GA. Automated system for gene annotation and metabolic pathway reconstruction using general sequence databases. Chem Biodivers. 2007:4(11):2593-602.

32. Lowe TM, Eddy SR. tRNAscan-SE: a program for improved detection of transfer RNA genes in genomic sequence. Nucleic Acids Res. 1997;25(5):955-64.

33. Lagesen $\mathrm{K}$, Hallin $\mathrm{P}$, Rodland EA, Staerfeldt HH, Rognes T, Ussery DW. RNAmmer: consistent and rapid annotation of ribosomal RNA genes. Nucleic Acids Res. 2007;35(9):3100-8.

34. Barrangou R, Fremaux C, Deveau H, Richards M, Boyaval P, Moineau S, et al. CRISPR provides acquired resistance against viruses in prokaryotes. Science. 2007;315(5819):1709-12.

35. Ruan $L, X u X$. Sequence analysis and characterizations of two novel plasmids isolated from Thermus sp. 4C. Plasmid. 2007;58(1):84-7.

36. Ebersbach G, Gerdes K. Plasmid segregation mechanisms. Annu Rev Genet. 2005;39:453-79.

37. Mooser D, Maneg O, MacMillan F, Malatesta F, Soulimane T, Ludwig B. The menaquinol-oxidizing cytochrome bc complex from Thermus thermophilus: protein domains and subunits. Biochim Biophys Acta. 2006;1757(9-10):1084-95.

38. Hellwig P, Soulimane T, Mantele W. Electrochemical, FT-IR and UVNIS spectroscopic properties of the caa3 oxidase from $T$. thermophilus. Eur J Biochem. 2002;269(19):4830-8.

39. Friedrich A, Prust C, Hartsch T, Henne A, Averhoff B. Molecular analyses of the natural transformation machinery and identification of pilus structures in the extremely thermophilic bacterium Thermus thermophilus strain HB27. Appl Environ Microbiol. 2002;68(2):745-55.

40. Averhoff B. Shuffling genes around in hot environments: the unique DNA transporter of Thermus thermophilus. FEMS Microbiol Rev. 2009;33(3):611-26.

41. Langille MG, Brinkman FS. IslandViewer: an integrated interface for computational identification and visualization of genomic islands. Bioinformatics. 2009;25(5):664-5.
42. Boyd EF, Almagro-Moreno S, Parent MA. Genomic islands are dynamic, ancient integrative elements in bacterial evolution. Trends Microbiol. 2009; 17(2):47-53.

43. Sorek R, Kunin V, Hugenholtz P. CRISPR-a widespread system that provides acquired resistance against phages in bacteria and archaea. Nat Rev Microbiol. 2008;6(3):181-6.

44. Grissa I, Vergnaud G, Pourcel C. The CRISPRdb database and tools to display CRISPRs and to generate dictionaries of spacers and repeats. BMC Bioinformatics. 2007;8:172.

45. Brock TD. Genus Thermus. In: N.R. Krieg and J.G. Holt (Eds). Bergey's Manual of Systematic Bacteriology, 1st ed Williams \& Wilkins Baltimore, MD. 1984;1:333-7.

46. Sandmann G. Carotenoid biosynthesis and biotechnological application. Arch Biochem Biophys. 2001;385(1):4-12.

47. Yokoyama A, Shizuri Y, Hoshino T, Sandmann G. Thermocryptoxanthins: novel intermediates in the carotenoid biosynthetic pathway of Thermus thermophilus. Arch Microbiol. 1996;165(5):342-5.

48. Armstrong GA, Alberti M, Hearst JE. Conserved enzymes mediate the early reactions of carotenoid biosynthesis in nonphotosynthetic and photosynthetic prokaryotes. Proc Natl Acad Sci U S A. 1990;87(24):9975-9.

49. Ohto C, Ishida C, Koike-Takeshita A, Yokoyama K, Muramatsu M, Nishino T, et al. Gene cloning and overexpression of a geranylgeranyl diphosphate synthase of an extremely thermophilic bacterium, Thermus thermophilus. Biosci Biotechnol Biochem. 1999;63(2):261-70.

50. Hoshino T, Fujii R, Nakahara T. Molecular cloning and sequence analysis of the crtB gene of Thermus thermophilus HB27, an extreme thermophile producing carotenoid pigments. Appl Environ Microbiol. 1993;59(9):3150-3.

51. Takaichi S, Mochimaru M. Carotenoids and carotenogenesis in cyanobacteria: unique ketocarotenoids and carotenoid glycosides. Cell Mol Life Sci. 2007;64(19-20):2607-19.

52. Bruggemann $\mathrm{H}$, Chen $\mathrm{C}$. Comparative genomics of Thermus thermophilus: Plasticity of the megaplasmid and its contribution to a thermophilic lifestyle. J Biotechnol. 2006;124(4):654-61.

53. Garrity GM, Lilburn TG, Cole JR, Harrison SH, Euzeby J, Tindall BJ. The Taxonomic Outline of Bacteria and Archaea version 7.7. Michigan State University Board of Trustees. 2007.

54. Bruno WJ, Socci ND, Halpern AL. Weighted neighbor joining: a likelihoodbased approach to distance-based phylogeny reconstruction. Mol Biol Evol. 2000;17(1):189-97.

55. Cole JR, Chai B, Farris RJ, Wang Q, Kulam-Syed-Mohideen AS, McGarrell DM, et al. The ribosomal database project (RDP-II): introducing myRDP space and quality controlled public data. Nucleic Acids Res. 2007;35(Database issue):D169-72.

56. Stothard P, Wishart DS. Circular genome visualization and exploration using CGView. Bioinformatics. 2005;21(4):537-9.

57. Field D, Garrity G, Gray T, Morrison N, Selengut J, Sterk P, et al. The minimum information about a genome sequence (MIGS) specification. Nat Biotechnol. 2008;26(5):541-7.

58. Weisburg WG, Giovannoni SJ, Woese CR. The Deinococcus-Thermus phylum and the effect of rRNA composition on phylogenetic tree construction. Syst Appl Microbiol. 1989;11:128-34.

59. Garrity GM, Holt JG. Bergey's Manual of Systematic Bacteriology. 2nd ed. New York: Springer; 2001

60. Validation of publication of new names and new combinations previously effectively published outside the IJSEM. International Journal of Systematic and Evolutionary Microbiology. Int J Syst Evol Microbiol. 2002;52:685-90.

61. Rainey FA, da Costa MS. Bergey's Manual of Systematic Bacteriology. 2nd ed. New York: Springer; 2001.

62. da Costa MS, Rainey FA. Bergey's Manual of Systematic Bacteriology. 2nd ed. New York: Springer; 2001.

63. Skerman VBD, MacGowan V, Sneath PHA. Approved Lists of Bacterial Names. Int J Syst Bacteriol. 1980;30:225-420.

64. Nobre MF, Trüper HG, da Costa MS. Transfer of Thermus ruber (Loginova et al. 1984), Thermus silvanus (Tenreiro et al. 1995), and Thermus chliarophilus (Tenreiro et al. 1995) to Meiothermus gen. nov. as Meiothermus ruber comb. nov., Meiothermus silvanus comb. nov., and Meiothermus chliarophilus comb. nov., respectively, and amendation of the genus Thermus. Int J Syst Bacteriol. 1996:46:604-6.

65. Ashburner M, Ball CA, Blake JA, Botstein D, Butler H, Cherry JM, et al. Gene ontology: tool for the unification of biology. The Gene Ontology Consortium. Nat Genet. 2000;25(1):25-9. 